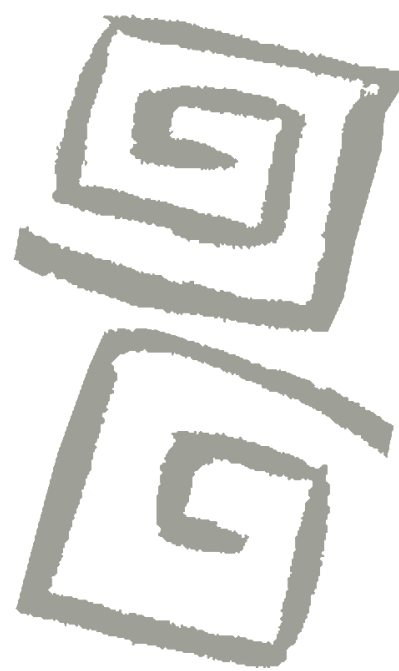

\title{
Estudio comparativo del estado nutricional de niños y niñas residentes en cuatro partidos de la provincia de Buenos Aires (Argentina), en el marco de la transición nutricional
}

\author{
Comparative study of the nutritional status of children \\ living in four counties of the province of Buenos Aires \\ (Argentina), in the context of nutrition transition
}

Evelia Edith Oyhenart ${ }^{1}$, María Fernanda Torres ${ }^{2}$, María Antonia Luis ${ }^{3}$, María Eugenia Luna ${ }^{4}$, Luis Eduardo Castro ${ }^{5}$, Mariela Garraza ${ }^{6}$, Bárbara Navazo María Cecilia Fucini ${ }^{8}$, Fabián Aníbal Quintero ${ }^{9}$, María Florencia Cesani ${ }^{10}$

'Doctora en Ciencias Naturales. Profesora Titular, Investigadora Principal, CONICET, Instituto de Genética Veterinaria, UNLP. Buenos Aires, Argentina. $\square$ (iD)

${ }^{2}$ Doctora en Ciencias Naturales. lefa de Trabajos Prácticos, Profesional Adjunto, CONICET, Instituto de Genética Veterinaria UNLP. Buenos Aires, Argentina. $\triangle$ (iD)

PProfesora Titular, Laboratorio de Investigaciones en Ontogenia y Adaptación, UNLP. Buenos Aires, Argentina. $\square$ (iD

${ }^{4}$ Doctora en Ciencias Naturales. Ayudante Diplomado, Instituto de Cenética Veterinaria UNLP. Buenos Aires, Argentina. $\square$ (iD)

${ }^{5}$ Doctor en Ciencias Naturales. Profesor Titular, Instituto de Genética Veterinaria, UNLP. Buenos Aires, Argentina. $\square$ (iD)

${ }^{6}$ Doctora en Ciencias Naturales. Ayudante Diplomado

Investigador Asistente CONICET, Instituto de Genética Veterinaria, UNLP. Buenos Aires, Argentina. $\triangle$ (iD)

${ }^{7}$ Doctoranda en Ciencias Naturales. Ayudante Diplomado, Becario CONICET, Instituto de Genética Veterinaria, UNLP. Buenos Aires, Argentina. $\square$ (iD)

${ }^{8}$ Odontóloga. Ayudante Diplomado, Facultad de Odontología, UNLP. Buenos Aires, Argentina. $\bowtie$ (iD)

${ }^{9}$ Doctor en Ciencias Naturales. Profesora Titular, Instituto de Genética Veterinaria. UNLP. Buenos Aires, Argentina. $\square$ (ID)

${ }^{10}$ Doctora en Ciencias Naturales. Jefe de Trabajos Prácticos, Investigador Independiente, CONICET, Instituto de Genética Veterinaria, UNLP. Buenos Aires, Argentina. $\square$ (ID)
RESUMEN El objetivo fue evaluar y comparar el estado nutricional en poblaciones infantojuveniles residentes en cuatro partidos de la provincia de Buenos Aires y observar si existen diferencias vinculadas a los contextos ambientales y socioeconómicos particulares, en el marco de la transición nutricional. Entre los ciclos lectivos 2008-2012, se relevó el peso y la talla y se calculó el índice de masa corporal de 7.873 escolares, entre 4 y 12 años de edad, residentes en los partidos de La Plata, Brandsen, Magdalena y Punta Indio. La información acerca del contexto de residencia se obtuvo mediante encuesta estructurada y autoadministrada. Alrededor del $40 \%$ de los escolares presentó malnutrición, y prevalecieron el sobrepeso en La Plata, la obesidad en Punta Indio y la desnutrición en Magdalena. El estado nutricional se vinculó con las condiciones socioambientales de residencia y dio cuenta de que estas poblaciones se ubican en distintas etapas del proceso de transición nutricional.

PALABRAS CLAVES Sobrepeso; Obesidad; Desnutrición; Condiciones Socioambientales; Escolares; Argentina.

\begin{abstract}
The aim of this study was to evaluate and compare the nutritional status of child populations living in four counties of the province of Buenos Aires to observe differences related to particular environmental and socioeconomic contexts in the framework of the nutrition transition. During the school years corresponding to the 20082012 period, weight and height were measured and body mass index was calculated for 7,873 school children between the ages of 4 and 12 years from the counties of La Plata, Brandsen, Magdalena and Punta Indio. Information about the contexts of residence was obtained using self-administered structured surveys. About $40 \%$ of schoolchildren presented malnutrition, with a prevalence of overweight in La Plata, obesity in Punta Indio and undernutrition in Magdalena. The nutritional status of the study populations was associated with the socioenvironmental conditions of residence, indicating that these populations are at different stages of the nutrition transition process.
\end{abstract}

KEY WORDS Overweight; Obesity; Undernutrition; Socio-environmental conditions; School children; Argentina. 


\section{INTRODUCCIÓN}

Se ha informado que, si bien a nivel global se ha registrado una disminución de la desnutrición entre los años 2014-2015, alrededor de 795 millones de personas continuaban sufriendo subnutrición ${ }^{(1)}$. Por otra parte, ya en el año 1998 la World Health Organization (WHO) declaró la obesidad como epidemia ${ }^{(2)}$. En efecto, se ha estimado que, en los últimos años, la prevalencia de exceso ponderal se ha duplicado y las cifras más recientes informaron que casi el $40 \%$ de la población adulta en el mundo padece sobrepeso y el $13 \%$ obesidad $^{(3)}$. Dicho aumento se ha explicado, fundamentalmente, como resultado de cambios en los hábitos alimentarios y de actividad física de las poblaciones. El reemplazo de dietas tradicionales por otras ricas en grasa, azúcar y alimentos industrializados, sumado al avance de la tecnología y al desarrollo de las ciudades modernas delinean el "ambiente obesogénico", en el cual los nuevos patrones de alimentación, trabajo, transporte y recreación hacen que las personas lleven una vida menos saludable y más sedentaria ${ }^{(4)}$.

Así, numerosas poblaciones padecen la doble carga de la malnutrición. La desnutrición y el exceso de peso pueden coexistir en una misma región, estrato social, familia y aún más, en una misma persona, como consecuencia de la deficiencia de micronutrientes que acompaña al exceso ponderal ${ }^{(5,6,7)}$. Esta paradoja se reconoce como una característica propia del proceso denominado "transición nutricional", que involucra un conjunto de cambios en los comportamientos alimentarios y estilos de vida de las poblaciones. Estos cambios promueven la tendencia al aumento del sobrepeso y de la obesidad y a ciertas patologías crónicas, como las cardiovasculares y la diabetes mellitus tipo II, en coexistencia con la desnutrición y otras enfermedades carenciales ${ }^{(8)}$. De esta manera, mientras que la coexistencia de desnutrición y obesidad caracteriza a las sociedades en transición nutricional, la prevalencia de sobrepeso y obesidad, como principal problema epidemiológico de malnutrición, caracteriza a las sociedades que estarían finalizando su transición ${ }^{(9)}$. En Argentina, la rápida urbanización, los cambios en la estructura demográfica y la incorporación de nuevas pautas de vida, junto con cambios culturales y en la accesibilidad a los alimentos, también promovieron, en las últimas décadas, importantes cambios en los patrones alimentarios y de actividad física ${ }^{(10)}$. En este sentido, un estudio realizado por Zapata et al. ${ }^{(11)}$ indicó que, en nuestro país, el consumo aparente de alimentos y bebidas se ha modificado entre 1996 y 2013, y registró un aumento en la adquisición de alimentos propios de los países industrializados y una reducción del consumo de alimentos tradicionales y con bajo nivel de industrialización, tales como vegetales y legumbres que, además, requieren mayor tiempo de elaboración.

No obstante, el proceso transicional no se produce del mismo modo en todas las poblaciones del territorio nacional, situación reflejada en las disparidades de prevalencias y tipos de malnutrición. Con relación a ello, en un estudio multicéntrico publicado en 2008 se registró una distribución clinal de la malnutrición infanto-juvenil, en el que las mayores prevalencias de desnutrición correspondían a las provincias del norte y las de sobrepeso y obesidad a las del sur ${ }^{(12)}$. Posteriormente, otros estudios nacionales dieron cuenta de que, en ciertos sectores de la población, las deficiencias nutricionales traducidas, principalmente, en desnutrición de tipo crónica y carencias de nutrientes específicos eran un problema vigente, aun cuando el sobrepeso y la obesidad habían adquirido una relevancia insospechada ${ }^{(13,14,15,16,17)}$.

Específicamente, en la provincia de Buenos Aires, estudios previos realizados por nuestro equipo de investigación permitieron caracterizar el estado nutricional de las poblaciones de La Plata, Punta Indio, Magdalena y Brandsen. Los resultados de dichas investigaciones mostraron que cada una de estas poblaciones, independientemente de la metodología empleada, estaban atravesando un proceso de transición nutricional ${ }^{(16,18,19,20)}$. En este sentido, el presente estudio tiene como objetivo evaluar y comparar el estado 
nutricional infanto-juvenil entre poblaciones residentes en cuatro partidos de la provincia de Buenos Aires y observar si existen diferencias vinculadas a los contextos ambientales y socioeconómicos particulares, en el marco de la transición nutricional.

\section{MATERIAL Y MÉTODOS}

\section{Zona de Estudio}

La provincia de Buenos Aires forma parte de la región pampeana y, dadas sus características climáticas y edafológicas, se caracteriza por ser una zona agrícola y ganadera por excelencia. Administrativamente, se divide en 135 municipios denominados partidos que, en términos de extensión geográfica, tamaño poblacional, tipo de actividad económica predominante y situación social, evidencian importantes diferencias ${ }^{(21)}$. En la Figura 1 se presenta el mapa de la provincia de Buenos Aires con su división político-administrativa y se indican los partidos considerados en el presente trabajo: La Plata, Brandsen, Punta Indio y Magdalena. Dichos partidos presentan características históricas, geográficas, demográficas, sociales y económicas particulares a la vez que semejantes.

El partido de La Plata, con una superficie de $926 \mathrm{~km}^{2}$ y una población de 654.324 habitantes ${ }^{(22)}$, constituye el centro administrativo provincial. Incluye, además del Ilamado "casco urbano", 18 localidades dependientes de una municipalidad central. Junto con el sector servicios, la administración pública y el sector industrial conforman las actividades económicas principales ${ }^{(22)}$.

Aproximadamente a 50 kilómetros de La Plata, se encuentra el partido de Brandsen, con una superficie de $1.130 \mathrm{~km}^{2}$ y una población de 26.352 habitantes $^{(22)}$, concentrada sobre todo en la ciudad cabecera del partido. Entre las actividades económicas más importantes se destacan las agrícolas, ganaderas y tamberas que, si bien en los últimos años se han visto notablemente reducidas, coexisten con actividades industriales vinculadas al agro y de servicios ${ }^{(22)}$.
Por su parte, Punta Indio y Magdalena, en comparación con La Plata y Brandsen, son partidos con baja densidad demográfica. Mientras que Punta Indio cuenta con una superficie de $1.627 \mathrm{~km}^{2}$ y una población de 9.362 habitantes, Magdalena posee una extensión de $1.863 \mathrm{~km}^{2}$ y una población de 16.603 habitantes. Ambos partidos se sustentan en una economía básicamente ganadera, contando además con tierras destinadas a la siembra de soja y de trigo ${ }^{(21,22)}$.

\section{Diseño del estudio y población}

El trabajo se deriva de un proyecto general que aborda el análisis del crecimiento, el estado nutricional y las enteroparasitosis infanto-juveniles en poblaciones urbanas $y$ rurales de diversas provincias de Argentina (PICT 01541). El presente estudio se llevó a cabo durante los ciclos lectivos 2008-2012,

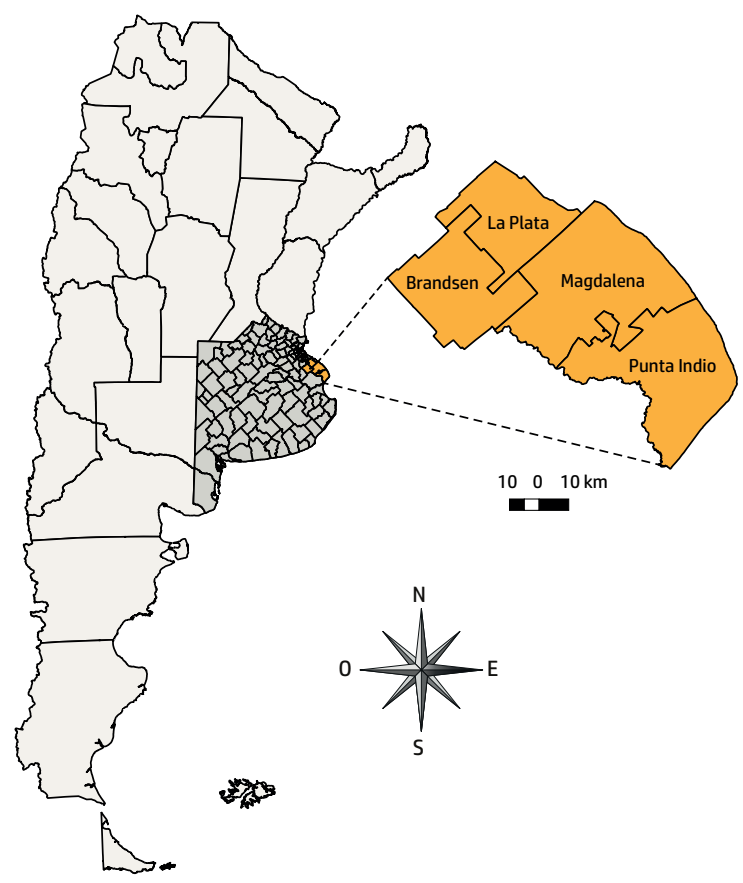

Figura 1. Ubicación geográfica de los partidos de La Plata, Brandsen, Magdalena y Punta Indio (Buenos Aires, Argentina).

Fuente: Elaboración propia. 
fue transversal e incluyó 7.873 escolares, de ambos sexos, con edades comprendidas entre 4 y 12 años, que asistían a establecimientos educativos públicos.

Para el cálculo del tamaño muestral se consideró la totalidad de los escolares correspondientes a los años de los ciclos lectivos en que fueron medidos. Los datos fueron aportados por la Dirección General de Escuelas de la provincia de Buenos Aires. Considerando un supuesto de varianza máxima $\left(p^{*} q=0,25\right)$ para distribución binomial, resolución del $3 \%$ y nivel de confianza del $95 \%$, el tamaño de la muestra requerido para La Plata fue de 1.053 escolares; Brandsen, 920; Punta Indio, 671; y Magdalena, 341.

La muestra relevada quedó conformada por 5.568 escolares de La Plata (2.791 niños y 2.777 niñas); 1.241 de Brandsen (590 niños y 651 niñas); 689 de Punta Indio (334 niños y 355 niñas) y 375 de Magdalena (185 niños y 190 niñas) superando el tamaño muestral mínimo requerido. Las escuelas fueron seleccionadas de manera no aleatoria a partir de un muestreo por conveniencia. Se consideraron escuelas públicas que figuraran en el listado de establecimientos dependientes de la Dirección General de Cultura y Educación, y que fueran autorizados para intervenir en el estudio. Sobre esta primera selección se optó por cubrir la representatividad del centro y la periferia de cada uno de los partidos y localidades que los integran. Se excluyeron los escolares con enfermedad manifiesta o indicación medicamentosa al momento del relevamiento, los que no contaban con autorización escrita de los padres o tutores y aquellos que, aun teniéndola, manifestaron su negativa a ser medidos.

El protocolo de este estudio fue aprobado por el Comité de Bioética de la Escuela Latinoamericana de Bioética CELABE. La investigación se desarrolló conforme a los principios proclamados en la Declaración Universal de los Derechos Humanos de 1948; las normas éticas instituidas por el Código de Nüremberg en 1947; la Declaración de Helsinski de 1964 y sus sucesivas enmiendas y clarificaciones; $y$ la Ley Nacional 25326, modificada por Ley 26343 de protección de datos personales y su Reglamentación № 1.558/01. El estudio incluyó tres ejes de análisis: antropométrico; ambiental y socioeconómico; y estadístico.

\section{Análisis antropométrico}

Siguiendo protocolos estandarizados ${ }^{(23)}$, se relevaron las siguientes variables: a) peso corporal (en kilogramos), empleando una balanza digital portátil (100g de precisión) que se calibró al inicio de cada sesión y en todos los casos los niños vistieron ropa liviana cuyo peso se descontó del peso total; y b) talla en centímetros utilizando un antropómetro vertical ( $1 \mathrm{~mm}$ de precisión) con el niño descalzo, erguido y con la cabeza orientada en el plano de Frankfort.

Con los datos de peso y talla se calculó el índice de masa corporal [IMC $=$ peso $(\mathrm{kg})$ / talla $\left(\mathrm{m}^{2}\right)$ ]. Los datos fueron estandarizados según z-scores, usando la referencia internacional $\mathrm{WHO}^{(24)}$. Las prevalencias de bajo peso para la edad, baja talla para la edad, bajo índice de masa corporal se determinaron tomando como punto de corte $<-2 z$, las de sobrepeso entre $1 z$ y $2 z$ y las de obesidad $>2 z$.

\section{Análisis ambiental y socioeconómico}

Se realizó una encuesta estructurada y autoadministrada a los padres o tutores de los escolares que participaron del estudio. Se incluyeron variables que comprendieron parámetros intra y peri domiciliarios tales como características constructivas de la vivienda, hacinamiento, forma de acceso al agua de consumo, eliminación de excretas, desagüe de aguas servidas a un sistema centralizado de colección de líquidos cloacales, red de distribución de energía eléctrica, red de distribución de gas natural, pavimento, servicio de recolección de residuos, y combustible utilizado para cocinar y calefaccionar. Adicionalmente, para complementar la información sobre el nivel socioeconómico familiar, se consideraron régimen de tenencia de la vivienda, nivel educativo y tipo de trabajo de padres y madres, cobertura médica mediante seguros de salud e 
ingresos familiares complementarios -incluyendo el acceso a planes sociales y/o programas alimentarios- la práctica de cultivo en huertas familiares y la cría de animales para autoconsumo(25).

\section{Análisis estadístico}

Se calcularon prevalencias para cada indicador de estado nutricional que fueron comparadas entre partidos mediante pruebas de chi cuadrado $\left(\chi^{2}\right)$. A su vez, los datos ambientales y socioeconómicos fueron analizados mediante análisis de componentes principales categóricos (ACPCat). Este método resulta apropiado para el tratamiento de datos multivariados de naturaleza heterogénea, como es el caso de las encuestas aplicadas que incluyen variables nominales, ordinales y multinominales. La técnica de ACPCat es eminentemente descriptiva y permite reducir la complejidad, sin perder información del conjunto de observaciones. En otras palabras, permite transformar un conjunto de variables intercorrelacionadas ( $p)$, en otro conjunto (q) de variables no correlacionadas $(q<p)$ Ilamadas componentes principales ${ }^{(25)}$. En este estudio, el ACPCat fue utilizado para reducir la dimensionalidad de las variables a dos componentes (dimensiones 1 y 2). En el espacio conformado por estas dimensiones se representan los vectores que denotan las correlaciones entre variables, permitiendo visualizar el rango de variación de las características ambientales y socioeconómicas de residencia de los niños.

Por último, se analizó la distribución de la población escolar de cada partido según los grupos generados por el ACPCat y se los comparó mediante pruebas de $\chi^{2}$.

\section{RESULTADOS}

Las prevalencias generales para cada indicador nutricional fueron: $4,0 \%$ de baja talla para la edad, 1,2\% de bajo peso para la edad, 0,8\% de bajo índice de masa corporal, $21,1 \%$ de sobrepeso y $11,4 \%$ de obesidad. Las mayores prevalencias de baja talla y bajo peso para la edad $(6,7 \%$ y $3,9 \%$, respectivamente) correspondieron a Magdalena, de sobrepeso a La Plata $(22,2 \%)$ y de obesidad a Punta Indio (14,1\%). A excepción del bajo índice de masa corporal, las diferencias entre partidos fueron significativas para todos los indicadores nutricionales (Tabla 1).

El ACPCat indicó, para los dos primeros componentes, un alfa de Cronbach del 0,90 y una varianza explicada del 29,3\%, probando buena fiabilidad del modelo ${ }^{(26)}$. En la Figura 2 se muestra cómo se ubican los vectores en el espacio conformado por las dos dimensiones del ACPCat. Este análisis nos permitió definir cuatro cuadrantes a partir de los cuales se

Tabla 1. Prevalencias de malnutrición por indicador y por partido. Provincia de Buenos Aires, Argentina (2008-2012).

$\begin{array}{lccccccc}\text { Indicador } & \begin{array}{c}\text { General } \\ (\%)\end{array} & \begin{array}{c}\text { Punta Indio } \\ (\%)\end{array} & \begin{array}{c}\text { Brandsen } \\ (\%)\end{array} & \begin{array}{c}\text { La Plata } \\ (\%)\end{array} & \begin{array}{c}\text { Magdalena } \\ (\%)\end{array} & \chi^{2} & \text { Valor de } p \\ \text { Baja talla para la edad } & 4,0 & 1,7 & 3,4 & 4,3 & 6,7 & 18,36 & 0,000 \\ \text { Bajo peso para la edad } & 1,2 & 1,0 & 0,9 & 1,2 & 3,9 & 16,28 & 0,000 \\ \text { Bajo índice de masa corporal } & 0,8 & 1,3 & 0,6 & 0,7 & 1,3 & 4,78 & 0,188 \\ \text { Sobrepeso } & 21,1 & 20,6 & 18,1 & 22,2 & 17,1 & 14,08 & 0,003 \\ \text { Obesidad } & 11,4 & 14,1 & 9,9 & 11,4 & 12,8 & 8,33 & 0,040 \\ \text { Fuente: Elaboración propia a partir de datos obtenidos del trabajo de campo. }\end{array}$

Salud Colectiva | Universidad Nacional de Lanús | ISSN 1669-2381 | EISSN 1851-8265 | doi: 10.18294/sc.2018.1576 
conformaron grupos con diferencias respecto a la calidad ambiental de residencia y al nivel socioeconómico familiar. A continuación, se describen las variables que definieron a cada grupo (Figura 2).

- Grupo 1: nivel educativo alto paterno y materno, cobertura de salud y trabajo formal paterno y materno.

- Grupo 2: disponibilidad de electricidad, gas natural por red, pavimento, eliminación de excretas mediante sistema cloacal, recolección de residuos y agua de consumo por red.

- Grupo 3: ayudas monetaria y alimentaria por parte del Estado y hacinamiento crítico.

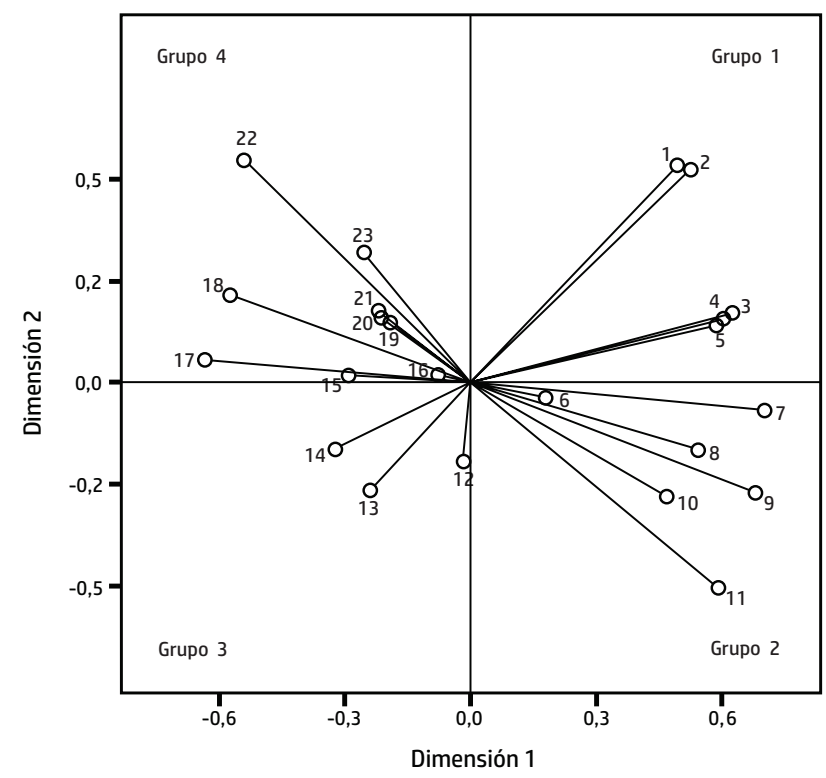

Figura 2. Análisis por componentes principales categóricos (ACPCat) de las variables ambientales y socioeconómicas. Provincia de Buenos Aires, Argentina (2008-2012).

Grupo 1: 1=Trabajo formal materno; $2=$ Trabajo formal paterno; 3=Nivel educativo materno; 4=Cobertura médica de salud; 5=Nivel educativo paterno. Grupo 2: 6=Red de energía eléctrica; 7=Gas natural; 8=Pavimento; 9=Eliminación de excretas mediante sistema cloacal; 10=Recolección de residuos; 11=Acceso al agua de consumo por red. Grupo 3: 12=Ayuda monetaria; 13=Ayuda alimentaria; 14=Hacinamiento crítico. Grupo 4: 15=Kerosene; 16=Material constructivo de la vivienda; 17=Garrafa; 18=Eliminación de excretas mediante pozo absorbente; 19=Régimen de tenencia de la vivienda; $20=$ Cría de animales; 21=Leña; $22=$ Acceso al agua de consumo por bomba; 23=Huerta.

Fuente: Elaboración propia a partir de datos obtenidos del trabajo de campo.
Tabla 2. Distribución de la población según grupos generados por análisis de componentes principales categóricos. Provincia de Buenos Aires, Argentina (2008-2012).

$\begin{array}{lcccc}\text { Localidad } & \text { Grupo } 1 & \text { Grupo } 2 & \text { Grupo } 3 & \text { Grupo } 4 \\ \text { Punta Indio } & 30,9 & 37,3 & 18,4 & 13,4 \\ \text { Brandsen } & 19,6 & 17,6 & 29,4 & 33,4 \\ \text { La Plata } & 20,1 & 22,0 & 28,6 & 29,3 \\ \text { Magdalena } & 22,7 & 27,2 & 30,1 & 20,0 \\ \text { Total } & 21,1 & 22,9 & 27,9 & 28,1\end{array}$

Fuente: Elaboración propia a partir de datos obtenidos del trabajo de campo.

- Grupo 4: uso de kerosene/garrafa/leña para cocinar/calefaccionar, material constructivo de la vivienda; eliminación de excretas mediante pozo absorbente; régimen de tenencia de la vivienda (prestada o cedida), provisión de agua para consumo por bomba, huerta y cría de animales.

La Tabla 2 muestra la distribución de la población de los distintos partidos en cada grupo definido por el ACPCat. Aproximadamente, el $68 \%$ de los escolares de Punta Indio se concentró en los grupos 1 y 2, el $63 \%$ de Brandsen y el $58 \%$ de La Plata lo hicieron en los grupos 3 y 4 y el $57 \%$ de Magdalena en los grupos 2 y 3. La comparación de dicha distribución mediante pruebas de $\chi^{2}$ indicó diferencias significativas $\left(\chi^{2}=297,63 ; p=0,0001\right)$.

\section{DISCUSIÓN Y CONCLUSIÓN}

A principio del siglo XXI, aproximadamente el $30 \%$ de la población mundial presentaba alguna forma de malnutrición en un contexto que permitía inferir que dicha situación iría en aumento ${ }^{(27)}$. Los resultados obtenidos en el presente estudio reafirman dicha tendencia, por cuanto se observó una prevalencia de malnutrición del orden del 38,5\%. Del total de escolares malnutridos, el 32,5\% tenía exceso ponderal, en tanto que el resto 
evidenció algún tipo de desnutrición, siendo el retardo lineal de crecimiento el más prevalente. Tal proporción y distribución de los indicadores de malnutrición mantuvieron vigencia conforme a lo hallado en investigaciones previas ${ }^{(16,18,19,20)}$.

El aumento sostenido del sobrepeso y la obesidad ha sido documentado en América Latina donde, a modo de ejemplo, el número de niños menores de 5 años aumentó de 5 a casi 6 millones en los últimos 25 años $^{(3)}$. Esta situación resulta un verdadero reto para la salud pública puesto que, además de los problemas propios de la obesidad, existen numerosas comorbilidades crónicas asociadas que comprometen la calidad de vida infantil presente y futura ${ }^{(28)}$. No obstante, el perfil nutricional en la región ha evolucionado de forma diferente, llevando a cada país a presentar distintos estadios de transición nutricional. Esto obedece a que el proceso transicional se desarrolla de forma simultánea o es precedido por cambios demográficos y epidemiológicos asociados a su vez con factores políticos, socioeconómicos y culturales propios de cada población ${ }^{(29)}$. En Sudamérica, los países que presentan mayor porcentaje de adultos obesos son Venezuela (30,8\%), Argentina $(29,4 \%)$ y Chile $(29,1 \%)$ indicando que se encuentran atravesando un estadio avanzado de la transición nutricional(30).

En nuestro país la transición se ha manifestado en varias regiones geográficas dando cuenta de su alcance generalizado. No obstante, las prevalencias de ambos extremos de la malnutrición han variado ${ }^{(13,14,15,16,18,19,20,31,32,33)}$. Coincidentemente, los resultados de este trabajo indicaron diferencias en el estado nutricional de los escolares de los cuatro partidos de la provincia de Buenos Aires. Así, la población de Magdalena registró los mayores porcentajes de desnutrición aguda y crónica, la de La Plata de sobrepeso y la de Punta Indio de obesidad. En Brandsen, en cambio, los indicadores nutricionales presentaron valores intermedios. Estos resultados indujeron a considerar que las poblaciones se encontrarían en distintos estadios de la transición debido, posiblemente, a las características ambientales y socioeconómicas propias de cada una. Con relación a ello, en el análisis de componentes principales, dos de los agrupamientos estuvieron definidos por variables socioeconómicas (grupos 1 y 3 ) mientras que los dos restantes lo hicieron por variables ambientales (grupos 2 y 4 ).

Las familias de Punta Indio presentaron, mayoritariamente, mejor nivel socioeconómico evidenciado por una mayor frecuencia de padres y madres con trabajo formalizado y alto nivel educativo y residentes en barrios con acceso a servicios públicos esenciales (grupos 1 y 2). Sin embargo, las familias de Magdalena se distribuyeron, principalmente, en el grupo 3 que correspondía a un ambiente más empobrecido, con altos porcentajes de hacinamiento y que recibían ayuda alimentaria y monetaria por parte del Estado. Las familias residentes en Brandsen, mayoritariamente, tenían estilos de vida con características atribuibles al ámbito rural, por cuanto empleaban bomba para extracción de agua subterránea para consumo, no disponían de cloacas para la eliminación de excretas, criaban animales y cultivaban vegetales para autoconsumo, entre otros (grupo 4). Por último, las familias de La Plata se distribuyeron, en su mayoría, en los grupos 3 y 4.

Probablemente, la situación "de bienestar" observada en Punta Indio fue la que generó condiciones propicias para que los niños y jóvenes dispusieran de mayor acceso a alimentos industrializados, procesados, con alto contenido energético, así como a bienes promotores de sedentarismo (TV, computadora, videojuegos, Internet, etc.) y la que condujo a que gran parte de ellos tuvieran exceso de peso. En contraposición, la población de Magdalena, ubicada en un contexto ambiental y socioeconómico menos favorable, presentó las prevalencias más altas de desnutrición en coexistencia con sobrepeso y obesidad. Estos resultados exhibirían una de las características de la transición nutricional: aumento del sobrepeso y la obesidad en contextos de pobreza. Al respecto, Aguirre ${ }^{(34)}$ refirió a los "gordos de la opulencia" a aquellos que, con ingresos suficientes, serían los principales consumidores de alimentos industrializados, golosinas, 
gaseosas, jugos azucarados, "snacks", etc. En tanto que los "gordos de la escasez", al tener restricción en las opciones de compra, tendrían una alimentación más monótona, basada principalmente en hidratos de carbono de alta densidad energética. Por último, las poblaciones de Brandsen y La Plata presentaron una situación nutricional intermedia. Posiblemente, la condición de ruralidad de algunos sectores de las poblaciones, antes mencionadas, habría facilitado el acceso a alimentos más saludables y no mercantilizados, ejerciendo efectos amortiguadores ante las dificultades alimentarias.

Los datos obtenidos permiten concluir que, aunque la desnutrición sigue siendo un problema vigente, en algunas poblaciones de la provincia de Buenos Aires, resulta evidente que el sobrepeso y la obesidad constituyen las alteraciones nutricionales más relevantes. El estado nutricional infanto-juvenil se vincula con las condiciones ambientales y socioeconómicas de residencia. Su variabilidad da cuenta de que estas poblaciones se ubican en distintas etapas del proceso de transición nutricional: la población de Punta Indio se encuentra en el extremo más avanzado, las de Brandsen y La Plata en un estadio intermedio, en tanto que la de Magdalena se ubica en una etapa más rezagada de dicho proceso.

\section{AGRADECIMIENTOS}

A los niños participantes, a sus padres y a las autoridades escolares. A la Sra. María Cristina Muñe, por la revisión general del manuscrito; a la Sra. Adriana Di Maggio, traductora pública nacional en lengua inglesa por la revisión idiomática; y al Sr. Omar Diego Magariños por la asistencia gráfica. A las entidades que financiaron esta investigación, la Agencia Nacional de Promoción Científica y Tecnológica (proyecto PICT 01541); la Universidad Nacional de La Plata (proyecto 11/N552) y Consejo Nacional de Investigaciones Científicas y Técnicas (proyecto PIP 112-200801-02197).

\section{REFERENCIAS BIBLIOGRÁFICAS}

1. Food and Agriculture Organization, International Fund for Agricultural Development, World Food Programme. The state of food insecurity in the world 2015: meeting the 2015 International Hunger Targets; taking stock of uneven progress [Internet]. Rome: Food and Agriculture Organization; 2015 [citado 10 ago 2017]. Disponible en: http://www.fao.org/3/a-i4646e.pdf.
2. World Health Organization. Obesity: preventing and managing the global epidemic [Internet]. Technical Report Series no. 894. World Health Organization; 1998 [citado 10 ago 2017]. Disponible en: https://tinyurl.com/jjwfqkz.

3. World Health Organization. Comisión para acabar con la obesidad infantil [Internet]. World Health Organization; 2016 [citado 21 dic 2017]. Disponible en: https://tinyurl.com/yakhg4kk.

4. Popkin BM. Relationship between shifts in food system dynamics and acceleration of the global nutrition transition. Nutrition Reviews. 2017;75(2): 73-82.

5. Rivera JA, Pedraza LS, Martorell R, Gil A. Introduction to the double burden of undernutrition and excess weight in Latin America. American Journal of Clinical Nutrition. 2014;100(6):S1613-S1616.

6. Galicia L, López de Romaña D, Harding KB, De-Regil LM, Grajeda R. Tackling malnutrition in Latin America and the Caribbean: challenges and opportunities. Revista Panamericana de Salud Pública. 2016;40(2):138-146. 
7. Torres-Roman JS, Azañedo D, Ruiz EF, Avilez JL, Málaga G. The double burden of malnutrition: a threat for Peruvian childhood. Gaceta Sanitaria. 2017;31(4):359-360.

8. Popkin BM. Nutrition transition and the global diabetes epidemic. Current Diabetes Reports. 2015; 15(9):64.

9. Rosique Gracia J, García AF, Villada Gómez BA. Estado nutricional, patrón alimentario y transición nutricional en escolares de Donmatías (Antioquía). Revista Colombiana de Antropología. 2012;48(1):97-124.

10. O’Donnell A, Carmuega E. La transición epidemiológica y la situación nutricional de nuestros niños. Boletín Centro de Estudios sobre Nutrición Infantil (CESNI). 1998;6:1-24.

11. Zapata ME, Rovirosa A, Carmuega E. Cambios en el patrón de consumo de alimentos y bebidas en Argentina, 1996-2013. Salud Colectiva. 2016;12(4):473-486.

12. Oyhenart EE, Dahinten SL, Alba JA, Alfaro EL, Bejarano IF, Cabrera GE, Cesani MF, Dipierri JE, Forte LM, Lomaglio DB, et al. Estado nutricional infanto juvenil en seis provincias de Argentina: variación regional. Revista Argentina de Antropología Biológica. 2008;10(1):1-62.

13. Ministerio de Salud de la Nación. La alimentación de los niños menores de 2 años: resultados de la Encuesta Nacional de Nutrición y Salud ENNyS 2010. Buenos Aires: Ministerio de Salud de la Nación; 2010 [citado 10 ago 2017]. Disponible en: https://tinyurl.com/y9gbbvbz.

14. Lomaglio DB. Evolution of height and nutritional status in school children between 1997 and 2010 in Antofagasta de la Sierra, Catamarca, Puna Argentina. High Altitude Medicine \& Biology. 2010;11(3):272.

15. Dahinten SL, Castro LE, Zavatti JR, Forte LM, Oyhenart EE. Growth of school children in different urban environments in Argentina. Annals of Human Biology. 2011;38(2):219-227.

16. Oyhenart EE, Garraza M, Bergel ML, Torres MF, Castro LE, Luis MA, Forte LM, Gamboa MI, Zonta ML, Cesani MF, et al. Caracterización del estado nutricional, enteroparasitosis y condiciones socio-ambientales de la población infanto-juvenil del partido de La Plata. Revista Argentina de Antropología Biológica. 2013;15(1):47-60.

17. Bergel Sanchís ML, Cesani MF, Oyhenart EE. Contexts of occurrence of child malnutrition in the district of Villaguay, Entre Ríos, Argentina: a multi- variate analysis. PLoS ONE. 2017;12(4):e0176346. doi: 10.1371/journal.pone.0176346.

18. Cesani MF, Garraza M, Bergel Sanchís ML, Luis MA, Torres MF, Quintero FA, Oyhenart EE. A comparative study on nutritional status and body composition of urban and rural schoolchildren from Brandsen district (Argentina). PLoS ONE. 2013;8(1):e52792. doi: 10.1371/journal. pone.0052792.

19. Bergel Sanchís ML, Quintero FA, Navazo B, Cesani MF, Garraza M, Torres MF, Luna ME, Luis MA, Castro LE, Oyhenart EE. Caracterización del estado nutricional en relación con factores socioambientales de la población escolar del Partido de Punta Indio (Provincia de Buenos Aires, Argentina). Revista Argentina de Antropología Biológica. 2016;18(2):1-13.

20. Torres MF, Luis MA, Garraza M, Oyhenart EE. Ruralidad y estado nutricional: un estudio en la población de Magdalena (provincia de Buenos Aires, Argentina). Revista del Museo de Antropología. 2017;10(1):77-86.

21. Quesada Aramburú J, Cadelli E. Hacia una clasificación de los municipios bonaerenses. Documento de Trabajo DPEPE $N^{\circ} 04 / 2012$. Buenos Aires: Ministerio de Economía Dirección Provincial de Estudios y Proyecciones Económicas; 2012.

22. Instituto Nacional de Estadística y Censos. Censo Nacional de Población, Hogares y Viviendas 2010 [Internet]. 2010 [citado 21 dic 2017]. Disponible en: https://www.indec.gov.ar/.

23. Lohman TG, Roche AF, Martorell R. Anthropometric standardization reference manual. Champaign: Human Kinetics Books; 1988.

24. World Health Organization, Multicentre Growth Reference Study Group. WHO Child growth standards: methods and development [Internet]. Geneva: World Health Organization; 2006 [citado 10 ago 2017]. Disponible en: https://tinyurl.com/ycgm5snd.

25. Oyhenart EE, Castro LE, Forte LM, Sicre ML, Quintero FA, Luis MA, Torres MF, Luna ME, Cesani MF, Orden AB. Socioenvironmental conditions and nutritional status in urban and rural schoolchildren. American Journal of Human Biology. 2008;20(4):399-405.

26. Peterson RA. A meta-analysis of Cronbach's coefficient alpha. Journal of Consumer Research. 1994;21(2):381-391.

27. Organización de las Naciones Unidas para la Agricultura y la Alimentación. El espectro de la malnutrición. En: Organización de las Naciones 
Unidas para la Agricultura y la Alimentación. El estado de la inseguridad alimentaria en el mundo [Internet]. Washington DC: FAO; 2000 [citado 10 ago 2017]. Disponible en: https://tinyurl.com/ y8kupzgm.

28. Thompson N, Mansfield B, Stringer M, Stewart B, Potter J, Fernengel K. An evidence-based resource for the management of comorbidities associated with childhood overweight and obesity. Journal of the American Association of Nurse Practitioners. 2016;28(10):559-570.

29. De La Cruz Sánchez EE. La transición nutricional: abordaje desde de las políticas públicas en América Latina. Opción. 2016;32(11):379-402.

30. Organización de las Naciones Unidas para la Agricultura y la Alimentación. Panorama de la inseguridad alimentaria en América Latina y el Caribe [Internet]. Roma: FAO; 2015 [citado 21 dic 2017]. Disponible en: http://www.fao.org/3/ai4636s.pdf
31. Meyer E, Carrillo R, Román EM, Bejarano IF Alfaro EL, Dipierri JE. Prevalencia de sobrepeso y obesidad en escolares jujeños de diferente nivel altitudinal según las referencias IOTF, CDC Y OMS. Archivos Argentinos de Pediatría. 2013;111(6):516-522.

32. Zonta ML, Oyhenart EE, Navone GT. Socioenvironmental variables associated with malnutrition and intestinal parasitoses in the child population of Misiones, Argentina. American Journal of Human Biology. 2014;26(5):609-616.

33. Brac J, Aimaretti N, Walz F, Martinelli M. Ingesta alimentaria, actividad física y estado nutricional de niños de dos localidades de Santa Fe con distinto grado de urbanización. Diaeta. 2014;32(146):6-13.

34. Aguirre P. La comida en los tiempos del ajuste. En: Torrado S, (dir.). El costo social del ajuste (Argentina 1976-2002). Buenos Aires: EDHASA; 2010. Tomo II. p. 51-102.

FORMA DE CITAR

Oyhenart EE, Torres MF, Luis MA, Luna ME, Castro LE, Garraza M, Navazo B, Fucini MC, Quintero FA, Cesani MF. Estudio comparativo del estado nutricional de niños y niñas residentes en cuatro partidos de la provincia de Buenos Aires (Argentina), en el marco de la transición nutricional. Salud Colectiva. 2018;14(3):597-606. doi: 10.18294/ sc. 2018.1576

Recibido: 25 de agosto de 2017 | Versión final: 15 de mayo de 2018 | Aprobado: 19 de junio de 2018

Este obra está bajo una licencia de Creative Commons Reconocimiento-NoComercial 4.0

Internacional. Reconocimiento - Permite copiar, distribuir y comunicar públicamente la obra.

A cambio, se debe reconocer y citar al autor original. No Comercial - Esta obra no puede ser

utilizada con finalidades comerciales, a menos que se obtenga el permiso.

http://dx.doi.org/10.18294/sc.2018.1576 